\title{
Effect of gastric decompression on postoperative vomiting in pediatric patients undergoing strabismus surgery: a randomized controlled study
}

Received May 20, 2019

Revised 1st, July 8, 2019

2nd, July 19, 2019

3rd, July 25, 2019

Accepted July 27, 2019

\section{Corresponding author}

Tae Hun An, M.D., Ph.D. Department of Anesthesiology and Pain Medicine, Chosun University Hospital, 365 Pilmun-daero, Donggu, Gwangju 61453, Korea Tel: 82-62-220-3223

Fax: 82-62-223-2333

E-mail: than@chosun.ac.kr ORCID

https://orcid.org/0000-0002-7405-0073

\section{Ki Tae Jung ${ }^{1,2}$, Se Hun Kim', Dong Joon Kim, ${ }^{1,2}$, Sang Hun Kim ${ }^{1,2}$, and Tae Hun $\mathrm{An}^{1,2}$}

Department of Anesthesiology and Pain Medicine, ${ }^{1}$ Chosun University Hospital, ${ }^{2}$ School of Medicine, Chosun University, Gwangju, Korea

\begin{abstract}
Background: Postoperative vomiting (POV) is one of the most serious complications in pediatric patients undergoing strabismus surgery. This study was conducted to test the hypothesis that gastric decompression (GD) could prevent POV caused by gastric distension after mask ventilation.

Methods: A total of 60 pediatric patients (ASA PS I-II, aged one to 10 years) were randomly allocated to two groups; Group $D(n=30)$ and Group $C(n=30)$. Induction of anesthesia was performed with careful face mask ventilation with $100 \% \mathrm{O}_{2}(3 \mathrm{~L} / \mathrm{min})$ and sevoflurane 3 vol\% to limit airway pressure below $20 \mathrm{cmH}_{2} \mathrm{O}$. Endotracheal intubation was done after confirming adequate neuromuscular blockade. Then, the patients in Group D received GD, while patients in Group C did not. After the surgery, POV was assessed during the emergence from anesthesia in the operating room and postanesthetic care unit (30 min and $60 \mathrm{~min}$ ).

Results: During the emergence, POV was significantly decreased in Group D compared to Group C (Group D 3.3\% vs. Group C 30.0\%, P = 0.006). The odds ratio analysis showed a lower incidence of POV in Group D (odds ratio $=0.080 ; 95 \%$ confidence limit: $0.009-0.685$ ) during the emergence period. There was no significant difference in the incidence of POV in the postanesthetic care unit (Group D 6.7\% vs. Group C $4.3 \%$ at 30 $\min , P=1.000 ; 0 \%$ in both groups at $60 \mathrm{~min}$ ).
\end{abstract}

Conclusions: GD reduced the incidence of POV in pediatric patients undergoing strabismus surgery during emergence.

Keywords: Anesthesia; Decompression; Pediatrics; Stomach; Strabismus; Vomiting.

\section{INTRODUCTION}

Postoperative vomiting (POV) is one of the most common and serious complications in pediatric surgical patients. The importance of POV in children has been emphasized because it is an important cause of morbidity in children [1]. Severe POV is associated, not only with physical complications, such as dehydration, electrolyte abnormalities, wound disruption, bleeding, or aspiration pneumonia, but also with delayed hospital discharge, the increased use of medical resources, increased health care costs, and decreased satisfaction [2]. Most of all, $\mathrm{POV}$ is a very unpleasant experience.

Strabismus surgery is an independent risk factor for POV in pediatric surgery [2-4]. Numerous efforts have been expend-

This is an Open Access article distributed under the terms of the Creative Commons Attribution Non-Commercial License (http://creativecommons.org/licenses/by-nc/4.0) which permits unrestricted non-commercial use, distribution, and reproduction in any medium, provided the original work is properly cited. 


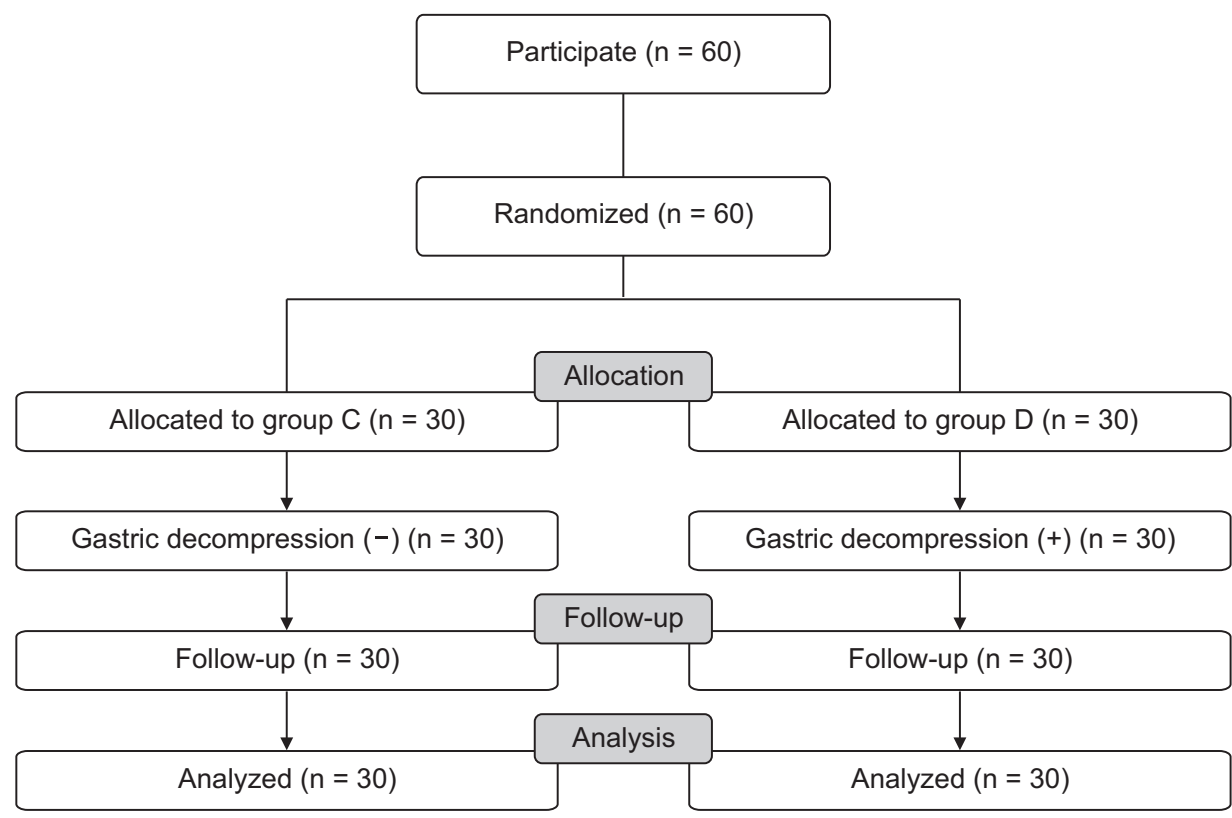

Fig. 1. Cohort flow chart. A total of 60 pediatric patients (American Society of Anesthesiologists physical status class I-II, aged one to 10 years) were randomly allocated into two groups. $\mathrm{Pa}$ tients in Group D ( $n=30)$ received gastric decompression after endotracheal intubation and patients in Group $\mathrm{C}(\mathrm{n}=$ 30) did not. At the end of the surgery, postoperative vomiting was assessed during anesthesia emergence (after extubation) and in the postanesthetic care unit (30 min and $60 \mathrm{~min}$ ) by a physician who did not participate in the anesthesia. ed to decrease POV associated with pediatric surgery according to their physiology and risk factors [1,2]. Many trials have been conducted to decrease POV by regulating anesthetic factors, such as premedications, selecting anesthetic agents, decreasing opioids, regulating intravenous fluids, and administering acupuncture or antiemetics $[1,2]$. However, other factors, such as gastric distension have been understudied.

Although controversial [5], gastric decompression (GD) has been shown to reduce POV after surgery [6]. Reducing gastric volume can decrease POV of gastrointestinal origin [7]. However, few studies have investigated the effect of GD on POV in pediatric patients. Children can be more susceptible to increased gastric volume during mask ventilation than adults because of anatomic differences [8].

Thus, we hypothesized, even though gentle mask ventilation during anesthesia induction can increase the gastric volume in children, decreasing gastric volume by GD could effectively reduce POV after pediatric strabismus surgery, which is one of the highest risk factors for POV (incidence up to $87 \%$ ) [4]. We conducted the study to evaluate the effect of GD on the prevention of POV after pediatric strabismus surgery.

\section{MATERIALS AND METHODS}

This prospective, randomized, controlled, double-blinded, parallel study was approved by the Institutional Review Board of University Hospital (no. 2015-11-002). A total of 60 pediatric patients, aged one to 10 years, scheduled for elective strabismus surgery at a single center were enrolled between February 1, 2016 and October 31, 2018 (Fig. 1). Patients with the following conditions were excluded: American Society of Anesthesiologists physical status class > III; history of previous POV; congenital diseases, such as hydrocephalus, tracheoesophageal fistula, or congenital heart disease; history of upper respiratory tract infection within two weeks; and respiratory disease, such as asthma. Written informed consent was obtained from the parents of all patients after a full explanation of the study. Patient characteristics, such as age, gender, height, weight, body mass index, American Society of Anesthesiologists physical status class, and duration of surgery, were assessed during the study.

The patients were randomly allocated to two groups using a random number table created by the computer. The patients in Group D $(\mathrm{n}=30)$ received GD immediately after endotracheal intubation and the patients in Group C ( $\mathrm{n}=$ 30) did not receive GD and acted as controls for the study. Both patients and investigators who assessed the POV were blinded to the allocation sequence.

Premedications were not administered to any patients. Upon arrival at the operating room, basal monitors, such as an electrocardiogram, pulse oximeter for oxygen saturation 
$\left(\mathrm{SpO}_{2}\right)$, non-invasive arterial pressure, and a neuromuscular transmission sensor (888418 M-NMT MechanoSensor, Datex-Ohmeda Inc., Finland) were placed. After cleaning the skin on the volar side of the wrist, surface electrodes were attached over the area of the ulnar nerve and sensors were placed on the index finger and thumb.

Anesthesia was induced and maintained by two physicians trained for more than three years. After administration of 5 $\mathrm{mg} / \mathrm{kg}$ thiopental sodium, mask ventilation was performed with $100 \% \mathrm{O}_{2}(3 \mathrm{~L} / \mathrm{min})$ plus sevoflurane $3 \mathrm{vol} \%$. During face mask ventilation, the adjustable pressure limiting valve was controlled to maintain the inspiratory airway pressure below $20 \mathrm{cmH}_{2} \mathrm{O}$. Manual mask ventilation was carefully performed to maintain the tidal volume measured on the volumeter at $8 \mathrm{ml} / \mathrm{kg}$ and to maintain the end-tidal $\mathrm{CO}_{2}$ between $35-40$ $\mathrm{mmHg}$.

Neuromuscular monitoring was started with an automatic search of stimulus current for the maximal response of the adductor pollicis muscle, which began with $10 \mathrm{~mA}$ stimulus and increased in steps of $5 \mathrm{~mA}$. When the response was no longer increased according to the increase in current, the supramaximal current was set as the current, which was automatically increased by $15 \%$ of the maximal current. If the supramaximal current was not found, $70 \mathrm{~mA}$ was set as the supramaximal current by the equipment and the supramaximal current was adjusted at $50 \mathrm{~mA}$. When the train-of-four count became less than 1 , intubation was performed with a Ring-Adair-Elwin (RAE) uncuffed endotracheal tube. The size of the RAE tube was selected using an age-based formula (age $/ 4+4$ or age $/ 4+4.5$ ) and the optimal tube size was confirmed by the air leakage test with a sustained inflation pressure of 20 to $25 \mathrm{cmH}_{2} \mathrm{O}$ [9].

A computer-generated random number table was used to assign patients to either Group C or D. The physician was informed of the group allocation by a nurse who was not part of the investigating team just before the endotracheal intubation. After the intubation, GD was performed immediately on the Group D patients but no GD was performed on the Group C patients. An 8 Fr nasogastric tube was carefully inserted into the stomach of the Group D patients. The depth of the gastric tube insertion was estimated by measuring from the external landmarks of the nose to the earlobe and to the xiphoid process (NEX method) and was confirmed by the aspiration of a small volume of gastric contents with a syringe
[10]. The nasogastric tube was connected to the wall-vacuum to regulate the suction pressure and GD was done carefully and intermittently with gentle pressing on the abdomen and the application of suction below $20 \mathrm{mmHg}$ to avoid gastric erosion [11].

Mechanical ventilation with an initial tidal volume of $8 \mathrm{ml} /$ $\mathrm{kg}$ was initiated and the tidal volume and frequency were adjusted to maintain the end-tidal $\mathrm{CO}_{2}$ between $35-40 \mathrm{mmHg}$. Anesthesia was maintained with sevoflurane and a $50 \% \mathrm{O}_{2}-$ air mixture. The end-tidal concentration of sevoflurane was adjusted to maintain the vital signs within $20 \%$ of their baseline values during the maintenance of anesthesia. No opioid or additional dose of neuromuscular blocking agent was used during the surgery.

At the end of the surgery, the administration of all anesthetics was discontinued and reversal agents (pyridostigmine 0.25 $\mathrm{mg} / \mathrm{kg}$ with glycopyrrolate $0.01 \mathrm{mg} / \mathrm{kg}$ ) were administered when the train-of-four ratio became more than 0.2. Extubation was performed when the train-of-four ratio became more than 0.9. The patients were transferred to the postanesthetic care unit (PACU) after emergence.

The primary outcome was the incidence of POV. To maintain study blindness, POV was assessed during the following three time intervals of anesthesia emergence by another physician who did not participate in the anesthesia: from the start of anesthesia arousal to transport to the PACU, during first $30 \mathrm{~min}$ in the PACU, and from 30 to $60 \mathrm{~min}$ in the PACU. Risk scores for postoperative vomiting for children were assessed according to the Eberhart classification. The scores ranged from 0 to 4 according to risk factors, such as age $>3$ years, duration of surgery $>30 \mathrm{~min}$, strabismus surgery, and history of POV [4]. Hemodynamic data, such as mean blood pressure and heart rate, were assessed before the induction (baseline), 1 min after GD, 1 min after extubation, and $60 \mathrm{~min}$ after extubation in the PACU.

The sample size was calculated using $\mathrm{G}^{*}$ Power3 free software (available at: http://www.psycho.uni-duesseldorf.de/ abteilungen/aap/gpower3). The effect size was 0.5 , which was a large effect size suggested by Cohen's conventional criteria because there were no previous studies on POV after GD in pediatric strabismus surgery patients [12]. Using $\alpha=$ 0.05 with a power of $80 \%$, the total sample size was calculated to be 52 . After assuming a $15 \%$ drop out rate, 30 patients were allocated to each group. 
Statistical analyses were performed using SPSS (IBM version 21, IBM Co., USA). The values are expressed as the mean (standard deviation) or the number of patients (\%). Normality of distribution was assessed with the KolmogorovSmirnov test and all the data showed normal distribution. The incidence of POV, the primary outcome of the study, was compared between the groups using the chi-squared test. Odds ratios (OR) and 95\% confidence intervals (95\% CI) were used to identify the correlations between GD and POV. Gender and American Society of Anesthesiologists physical status class were analyzed by the chi-squared test. Age, risk score, weight, height, body mass index, and duration of surgery were analyzed by $t$-tests. Hemodynamic changes were analyzed by repeat measures two-way ANOVA and $t$-tests was used to analyze differences between the groups. Post-hoc tests were conducted with Turkey's honestly significant difference test. $\mathrm{P}<0.05$ was considered statistically significant.

\section{RESULTS}

A total of 60 patients were assessed for eligibility and enrolled in the study. There were no significant differences in the characteristic data, risk scores for postoperative vomiting, or the duration of surgery between the groups (Table 1).

The incidence of POV differed during emergence (Table 2). During anesthesia emergence, POV developed in only $3.3 \%$ of the patients in Group D, while 30\% of the patients developed POV in Group C ( $\mathrm{P}=0.006)$. The odds ratio analysis showed a lower incidence of POV in Group D (OR $=0.080$; 95\% CI: 0.009-0.685) than in Group C during the emergence period. However, there were no significant differences in the incidence of POV between Group C and D in the PACU. There were also no significant differences in the hemodynamic data assessed at various times (Table 3).

\section{DISCUSSION}

In the current study, GD reduced the incidence of POV during emergence (3.3 vs. $30.0 \%$ in control) in pediatric patients undergoing strabismus surgery.

POV is a common complication after pediatric surgery and can result in several types of complications. POV in children is an important cause of morbidity and a rational approach is needed to minimize this unpleasant complication [1].

Table 1. Patient Characteristics

\begin{tabular}{|c|c|c|c|}
\hline Variable & Group C $(n=30)$ & Group D $(n=30)$ & $P$ value \\
\hline Age (yr) & $5.9 \pm 2.1(5.11-6.11)$ & $6.2 \pm 2.4(5.32-6.97)$ & 0.555 \\
\hline Age group & & & 1.000 \\
\hline$<3 y r$ & $2(6.7)$ & $2(6.7)$ & \\
\hline$\geq 3 \mathrm{yr}$ & $28(93.3)$ & $28(93.3)$ & \\
\hline Risk score & $2.93 \pm 0.25$ & $2.93 \pm 0.25$ & 1.000 \\
\hline Gender (F/M) & $20 / 10$ & $19 / 11$ & 1.000 \\
\hline Height (cm) & $117.3 \pm 12.7(112.87-121.68)$ & $117.7 \pm 15.8(111.97-122.80)$ & 0.921 \\
\hline Weight (kg) & $23.5 \pm 6.2(21.26-25.58)$ & $25.2 \pm 10.4(21.53-28.84)$ & 0.446 \\
\hline Body mass index & $16.7 \pm 2.1(16.00-17.48)$ & $17.3 \pm 3.2(16.27-18.48)$ & 0.370 \\
\hline ASA PS class (I/II) & $30 / 0$ & $29 / 1$ & 1.000 \\
\hline Duration of surgery (min) & $54.1 \pm 18.0(47.67-60.19)$ & $58.9 \pm 16.4(52.77-64.16)$ & 0.291 \\
\hline
\end{tabular}

Values are presented as mean \pm SD (95\% confidence interval) or number (\%). Group C, without gastric decompression as a control; Group D, with gastric decompression; Risk score, assessed by Eberhart classification [4]. ASA PS: American Society of Anesthesiologists physical status.

Table 2. The Incidence of Postoperative Vomiting

\begin{tabular}{|c|c|c|c|c|}
\hline Time interval & $\begin{array}{l}\text { Group C } \\
(n=30)\end{array}$ & $\begin{array}{l}\text { Group D } \\
(n=30)\end{array}$ & P value & $\begin{array}{c}\text { Odds ratio } \\
\text { (95\% confidence interval) }\end{array}$ \\
\hline During emergence & $9(30.0)$ & $1(3.3)$ & 0.006 & $0.080(0.009-0.685)$ \\
\hline During $30 \mathrm{~min}$ in the PACU & $2(6.7)$ & $1(4.3)$ & 1.000 & $0.483(0.041-5.628)$ \\
\hline From $30 \mathrm{~min}$ to $60 \mathrm{~min}$ in the PACU & $0(0)$ & $0(0)$ & - & - \\
\hline
\end{tabular}

Values are presented as number (\%). Group D, Group C, without gastric decompression as a control; Group D, with gastric decompression. POV: postoperative vomiting, PACU: postanesthetic care unit. 
Table 3. Hemodynamic Changes over Time

\begin{tabular}{|c|c|c|c|c|}
\hline & Time interval & Group C $(n=30)$ & Group $D(n=30)$ & $P$ value \\
\hline \multirow{4}{*}{$\begin{array}{c}\text { Mean blood } \\
\text { pressure } \\
\text { (mmHg) }\end{array}$} & Baseline (before induction) & $82.7 \pm 14.6(77.46-87.47)$ & $79.9 \pm 10.5(76.27-84.24)$ & 0.331 \\
\hline & 1 min after gastric decompression & $93.9 \pm 14.3(88.73-98.79)$ & $97.2 \pm 17.8(91.36-103.81)$ & 0.179 \\
\hline & 1 min after extubation & $97.2 \pm 15.1(91.83-102.32)$ & $96.0 \pm 15.3(90.81-101.36)$ & 0.753 \\
\hline & PACU (30 min after extubation) & $80.6 \pm 10.9(76.55-84.88)$ & $79.3 \pm 10.8$ (75.72-83.15) & 0.700 \\
\hline \multirow{4}{*}{$\begin{array}{l}\text { Heart rate } \\
\text { (beats/min) }\end{array}$} & Baseline (before induction) & $105.5 \pm 14.4(100.70-111.20)$ & $109.0 \pm 23.2(101.03-117.55)$ & 0.110 \\
\hline & $1 \mathrm{~min}$ after gastric decompression & $120.2 \pm 12.8(115.45-124.93)$ & $123.1 \pm 14.7(118.08-128.50)$ & 0.212 \\
\hline & 1 min after extubation & $131.6 \pm 13.6(125.92-136.68)$ & $129.1 \pm 19.9(121.73-135.92)$ & 0.135 \\
\hline & PACU (30 min after extubation) & $96.5 \pm 17.8(90.42-102.97)$ & $105.7 \pm 21.1(98.57-113.34)$ & 0.228 \\
\hline
\end{tabular}

Values are presented as mean \pm SD (95\% confidence interval). Group C, without gastric decompression as a control; Group D, with gastric decompression. PACU: postanesthetic care unit.

Children also perceive postoperative nausea and vomiting as distress and loathing as adults [13]. Although the physiology of emesis is a complicated response mediated by the emetic center of the brain in the lateral reticular formation of the medulla [2], no drug can completely prevent POV [13]. Thus, indiscriminate prophylaxis by antiemetic agents is unnecessary and identifying the risk factors for POV in pediatric patients and applying targeted prophylactic therapy has benefits [14].

According to previous reports, the incidence of POV is about 13 to $42 \%$ in all pediatric patients [2]. The known risk factors of POV in pediatric patients include patient factors, surgical factors, and anesthetic factors $[1,2]$. Patient factors, such as age, history of POV, and gender, and surgical factors, such as duration of surgery and type of surgery, are beyond the anesthesiologist's control. However, the anesthesiologist can prepare antiemetic prophylaxis by assessing those risk factors. A study investigating the risk of POV in pediatric patients suggested four independent risk factors: duration of surgery $\geq 30 \mathrm{~min}$, age $\geq 3$ years, strabismus surgery, and history of POV (or familial history of postoperative nausea and vomiting) [4]. According to the Eberhart classification scoring system, the incidence of POV was 9, 10, 30, 55, and $70 \%$ for risk factors scored as $0,1,2,3$, and 4, respectively [4].

Some risk factors were controlled during the conduct of the study. Patient factors were controlled by exclusion criteria (history of POV) and randomization during enrollment. Unfortunately, we enrolled children under three years of age due to the epidemiology of strabismus (surgery for congenital strabismus is conducted before patients are three years old). However, there were only two patients aged under three years in each group and there was no significant difference between the groups in the patient characteristics (history of POV, age, and female gender). In the surgical factors, the duration of surgery in both groups was about one hour and there was no significant difference between the groups. We also controlled anesthetic factors by avoiding opioid administration during surgery. However, sevoflurane and anticholinergics were used for the maintenance of anesthesia and reversal of the neuromuscular blockade. As a result, the patients in the current study had the same high-risk factors (strabismus surgery, duration of surgery more than $30 \mathrm{~min}$, age, and use of volatile anesthetics and anticholinergics) but there are no significant differences between the groups. We assessed the risk scores for postoperative vomiting for children and the mean score of the patients of the current study was 2.93. There was no difference in the risk scores between the groups. According to the Eberhart classification scoring system, the predicted incidence of POV with a risk score 3 is about $55 \%$ [4]. However, $30 \%$ of the patients who did not receive GD in the study showed POV.

In the current study, we tried GD as antiemetic prophylaxis instead of using prophylactic antiemetics. We hypothesized that gas inspired into the stomach during mask ventilation could cause vomiting. Gastric distention can develop despite gentle face mask ventilation with inspiratory pressure below the esophageal sphincter pressure (mean $30.6 \mathrm{mmHg}$ ) in children older than one year of age [15]. According to a study of Lee et al. [16], the median inspiratory pressure inducing gastric insufflation was $18 \mathrm{cmH}_{2} \mathrm{O}$ in pediatric patients and the authors suggested limiting the inspiratory pressure to less than $12-14 \mathrm{cmH}_{2} \mathrm{O}$ to minimize gastric insufflation. Another study by Lee et al. [17] showed that the incidence of gastric insufflation was $48 \%$ after manual face mask ventilation with a mean peak airway pressure of $16.1 \mathrm{cmH}_{2} \mathrm{O}$. Thus, the conventional face mask ventilation that limited inspiratory pressure to less than $20 \mathrm{cmH}_{2} \mathrm{O}$ used in this study should induce 
gastric distension.

Vagal neurocircuitry plays an important role in the provocation of nausea and vomiting. Nucleus tractus solitarius (NTS) is the recipient of direct or indirect sensory input from the abdominal and thoracic vagus nerves, pharynx, trigeminal nerve, spinal tract, and cerebellum [18]. The NTS regulates the medullary reflexes in the generation of nausea and vomiting. Distention of the stomach increases activation of mechanosensitive vagal afferents and the distention-evoked vagal afferents encode noxious information and transmit painful stimuli to the NTS. Thus, gastric inflation during mask ventilation activates mechanoreceptors, sending afferent signals via the vagus nerve, which may result in vomiting $[18,19]$. Therefore, there is a possibility that inspired gas into the stomach during mask ventilation can evoke vomiting by gastric distension.

A recent study showed that GD reduced the incidence and severity of POV in ear, nose, and throat (ENT) surgery [6]. Erkalp et al. [6] suggested that GD was a way to minimize POV factors and the number of studies on prophylactic GD should be increased in different surgeries because it can be a viable alternative for pharmacological treatment for POV. These suggestion agree with our results.

In contrast to our study, some previous studies have shown that GD did not decrease POV [20] but increased the risk of POV [21]. However, these previous studies were conducted on adult populations, not on pediatric patients. The capacity of the stomach in children is much smaller than that in adults [8]. Thus, even a small volume of inspired gas can be enough to generate a vomiting response. Still, there are only two studies on the effect of GD on POV after pediatric surgery and they concluded that GD did not decrease the incidence of POV [22,23]. However, those studies assessed POV after tonsillectomy, not strabismus surgery. Moreover, both studies used opioids, which is one of the highest risk factors for POV during anesthesia. In contrast, our study was conducted on patients undergoing strabismus surgery, which is an independent risk factor for POV with an incidence higher than other surgeries [4] and opioids were not used during the surgeries. These discrepancies would reveal the difference in outcomes following the use of GD as antiemetic prophylaxis.

Although recent consensus guidelines for the management of postoperative nausea and vomiting suggested that routine gastric decompression is ineffective in decreasing POV in children, the conclusion may have been based on insufficient evidence [5]. However, prophylactic therapy targeted to the identified risk factors of POV in pediatric patients is important [14]. An early review also suggested that gastric suction may be a valuable method of reducing vomiting by gastrointestinal origin [7]. Regrettably, the number of studies on the effect of GD on POV is still much smaller than the number of pharmacologic treatments. GD is an inexpensive and easy method to decrease POV in pediatric ENT surgery with few complications [6]. More well-controlled research on the effect of GD on POV is required to confirm the effectiveness and increase the data on this technique.

There were several limitations to the current study. First, we could not control all the risk factors of POV in the study protocol. Using total intravenous anesthesia and sugammadex for the reversal of neuromuscular blockade would be better ways to control the risk factors of POV but the use of propofol and sugammadex in pediatric patients is not still approved in Korea. However, we tried to maintain identical exposure to risk factors in both groups and there was no significant difference in the risk factors. Second, we only assessed early vomiting for about an hour. Pediatric POV can last for about a day, therefore, POV should be assessed at early (0-6 h) and later (6-24 h) postoperative periods [2]. Unfortunately, we could not assess later POV because most of the patients were discharged about four to six hours after surgery and this could be an important limitation of the current study. Third, we were unable to quantify the volume of the gastric contents. The amount of aspirated gastric content is associated with the severity of POV [6]. However, we used wall-vacuum suction to keep the pressure low (less than $20 \mathrm{mmHg}$ ) and avoid gastric erosion. We also conducted GD immediately after intubation and most of the gastric contents were only gases. Thus, we could not assess the amount of aspirated gastric content.

In conclusion, GD effectively reduced the incidence of POV during anesthesia emergence in pediatric patients undergoing strabismus surgery. GD can be a simple method for POV prophylaxis after pediatric strabismus surgery. Further research investigating the effectiveness of GD according to different POV risk factors is needed. 


\section{ACKNOWLEDGMENTS}

This study was supported by research fund from Chosun University Hospital, 2019.

\section{CONFLICTS OF INTEREST}

No potential conflict of interest relevant to this article was reported.

\section{ORCID}

Ki Tae Jung, https://orcid.org/0000-0002-2486-9961

Se Hun Kim, https://orcid.org/0000-0002-3603-7740

Dong Joon Kim, https://orcid.org/0000-0002-3072-4734

Sang Hun Kim, https://orcid.org/0000-0003-3869-9470

\section{REFERENCES}

1. Munro H. Postoperative nausea and vomiting in children. J Perianesth Nurs 2000; 15: 401-7.

2. Rose JB, Watcha MF. Postoperative nausea and vomiting in paediatric patients. Br J Anaesth 1999; 83: 104-17.

3. Tramèr $M$, Moore A, McQuay $H$. Prevention of vomiting after paediatric strabismus surgery: a systematic review using the numbers-needed-to-treat method. Br J Anaesth 1995; 75: 556-61.

4. Eberhart LH, Geldner G, Kranke P, Morin AM, Schäuffelen A Treiber $\mathrm{H}$, et al. The development and validation of a risk score to predict the probability of postoperative vomiting in pediatric patients. Anesth Analg 2004; 99: 1630-7.

5. Gan TJ, Diemunsch P, Habib AS, Kovac A, Kranke P, Meyer TA, et al. Consensus guidelines for the management of postoperative nausea and vomiting. Anesth Analg 2014; 118: 85-113.

6. Erkalp K, Kalekoglu Erkalp N, Sevdi MS, Korkut AY, Yeter H, Ege SS, et al. Gastric decompression decreases postoperative nausea and vomiting in ENT surgery. Int J Otolaryngol 2014; 2014: 275860 .

7. Palazzo MG, Strunin L. Anaesthesia and emesis. I: etiology. Can Anaesth Soc J 1984; 31: 178-87.

8. Scammon RE. Some graphs and tables illustrating the growth of the human stomach. Am J Dis Child 1919; 17: 395-422.

9. Kim SH, An TH. Oral endotracheal intubation in pediatric anesthesia. Anesth Pain Med 2018; 13: 241-7.
10. Farrington M, Lang S, Cullen L, Stewart S. Nasogastric tube placement verification in pediatric and neonatal patients. Pediatr Nurs 2009; 35: 17-24.

11. Lewis SM, Bucher L, Heitkemper MM, Harding M, Kwong J, Roberts D. Medical surgical nursing: assessment and management of clinical problems. St. Louis, Elsevier. 2017, p 1104.

12. Cohen J. A power primer. Psychol Bull 1992; 112: 155-9.

13. Gan T, Sloan F, Dear Gde L, El-Moalem HE, Lubarsky DA. How much are patients willing to pay to avoid postoperative nausea and vomiting? Anesth Analg 2001; 92: 393-400.

14. Scuderi PE, James RL, Harris L, Mims GR 3rd. Antiemetic prophylaxis does not improve outcomes after outpatient surgery when compared to symptomatic treatment. Anesthesiology 1999; 90: 360-71.

15. Moroz SP, Espinoza J, Cumming WA, Diamant NE. Lower esophageal sphincter function in children with and without gastroesophageal reflux. Gastroenterology 1976; 71: 236-41.

16. Lee JH, Jung H, Kim EH, Song IK, Kim HS, Kim JT. Optimal inspiratory pressure for face mask ventilation in paralyzed and unparalyzed children to prevent gastric insufflation: a prospective, randomized, non-blinded study. Can J Anaesth 2018; 65: 128895.

17. Lee JH, Jung H, Jang YE, Kim EH, Song IK, Kim HS, et al. Manual vs pressure-controlled facemask ventilation during the induction of general anesthesia in children: A prospective randomized controlled study. Paediatr Anaesth 2019; 29: 331-7.

18. Babic T, Browning $\mathrm{KN}$. The role of vagal neurocircuits in the regulation of nausea and vomiting. Eur J Pharmacol 2014; 722: 38-47.

19. Rabey PG, Smith G. Anaesthetic factors contributing to postoperative nausea and vomiting. Br J Anaesth 1992; 69(7 Suppl 1): 40S-45S.

20. Hovorka J, Korttila K, Erkola O. Gastric aspiration at the end of anaesthesia does not decrease postoperative nausea and vomiting. Anaesth Intensive Care 1990; 18: 58-61.

21. Trépanier CA, Isabel L. Perioperative gastric aspiration increases postoperative nausea and vomiting in outpatients. Can J Anaesth 1993; 40: 325-8

22. Chukudebelu O, Leonard DS, Healy A, McCoy D, Charles D, Hone S, et al. The effect of gastric decompression on postoperative nausea and emesis in pediatric, tonsillectomy patients. Int J Pediatr Otorhinolaryngol 2010; 74: 674-6.

23. Jones JE, Tabaee A, Glasgold R, Gomillion MC. Efficacy of gastric aspiration in reducing posttonsillectomy vomiting. Arch Otolaryngol Head Neck Surg 2001; 127: 980-4. 